\title{
Truth and Beauty in Physics and Biology
}

Ben D. MacArthur ${ }^{1,2,3}$

${ }^{1}$ The Alan Turing Institute, London, UK

${ }^{2}$ Mathematical Sciences, University of Southampton, Southampton, UK

${ }^{3}$ Centre for Human Development, Stem Cells and Regeneration, Faculty of Medicine, University of Southampton, Southampton, UK

Email: bdm@soton.ac.uk

\section{Physicists and biologists have different conceptions of beauty. A better appreciation of these differences may bring the disciplines closer and help develop a more integrated view of life.}

When I was a child, I was fascinated by the equations in my father's physics textbooks (he was a physics teacher and is an avid reader). I knew nothing of physics when I first saw these equations, but I sensed that they were important, and their mystery was attractive. But it was not just their mystery that drew me to them. I was also taken by the visual form of the equations themselves. To me, they had an abstract appeal of their own and - whatever they represented - they were works of art. I found them beautiful.

Now that I have studied mathematics and physics myself, I know more about what many of these equations mean, and the deeper beauty of the ideas that they convey. Yet, I still find myself attracted to their visual form; I still like the way they look. Some equations have a pleasing balance and mirror the elegance of the notions they represent in their visual aesthetic.

I don't think that this attraction is entirely superficial. It represents an appreciation of form that reflects something of the human response to the character of the natural world, and I am sure that many others feel it too. Appreciation of the beauty of the ideas that great equations represent, the physical or abstract world they describe, and their visual form are not exclusive. They are different aspects of their inherent beauty that are interrelated and complement each other. Beauty and truth have many facets.

In a 2014 study of the neurobiology of the appreciation of mathematical beauty, a group of mathematicians were shown a series of formulae and asked to judge their 'beauty' [1]. Each participant's brain activity was monitored using functional magnetic resonance imaging while performing this task, to identify the areas of the brain that became active when mathematical beauty was perceived. Remarkably, appreciation of mathematical beauty was associated with activity of field A1 of the medial orbito-frontal cortex, an emotional area of the brain that is associated with perception of beauty in other sources (such as the visual arts and music). Notably, the equations presented were a sample from across the mathematical and physical sciences - from number theory to mathematical biology, including both well-known and obscure equations - and so the participants did not necessarily understand them all. Although there was a positive association between comprehension and appreciation of beauty, this association was imperfect. Some candidates identified as beautiful equations they did not fully understand. Some participants from a control group of non-mathematicians did the same.

These observations indicate that the perception of mathematical beauty is not entirely contingent on intellectual comprehension. It is not clear why this is the case. One possibility is that beautiful equations have a balanced form that is neither too banal nor too complex that suggests something of deep importance and, whether they are fully comprehended or not, they impart a positive feeling of awe - of being part of something large and mysterious but not overwhelming. I think that something like this drew me to equations as a child: I was unconsciously aware that, although I did not understand them, someone else did and so their presence implied that the world makes sense, and their mystery was therefore both exciting and comforting rather than unsettling. This possibility concords with the view that aesthetic judgments are "expressions of our feeling that something makes sense to us" [2]. We dislike too much simplicity because it is boring; we dislike too much complexity because it seems to imply incomprehensibility. 


\section{Beauty in physics and biology}

Physicists often seek unifying laws or theories that are able to concisely explain experimental observations. Elegance, or simplicity, is frequently cited as a guiding principle in this search, and many physicists see beauty in simplicity $[3,4,5]$. This is not just a matter of parsimony (given a range of explanations, you should choose the simplest), but rather represents a deeply held belief - arguably a foundational belief, or axiom that although the world may seem complex, the truth is simple, elegant, harmonious. Richard Feynman famously summarized this view in his lectures on the character of physical law: "You can recognize truth by beauty and simplicity ... inexperienced students, make guesses that are very complicated. And it sort of looks like it's alright. But I know that's not true because the truth always turns out to be simpler than you thought" [3]. His zeal is inspiring, but the implication is clear: if you think that the world is complex then you have not yet understood it properly.

This view is not without basis. Some of the great discoveries in physics have been fundamentally guided by a faith in beauty as a guide. Murray Gell-Mann told of how, in 1957, he and his colleagues dared to propose a new theory for the weak force that contradicted extensive experimental evidence "because we figured it was so beautiful, it's gotta be right!" [4]. This was a bold proposition: to use beauty as a guide to truth is one thing; to suggest that it is a better guide than empirical observation is another altogether. Yet, remarkably, the experiments were wrong, and they were right. Activity deep within the medial orbito-frontal cortex of Gell-Mann's brain gave rise to insight about the fundamental structure of the universe. His appreciation of mathematical beauty, and conviction that this emotion should be trusted in spite of the evidence, helped us understand the world better. Beauty was a more reliable guide than empiricism.

Not all scientists see things this way, however.

Because life is so enormously complex, biologists, by contrast, often focus on particulars and apparently have less concern for generalizations or notions of beauty or elegance. At first, this seems to imply a lack of imagination that reduces biology to mere "stamp collecting" [6]. However, this caricature is unfair. The biologist's preference for specifics is not due lack of aesthetic sense: the best biology is equal in imagination, creativity and insight to the best physics. Rather, it stems from an alternative perspective on science, beauty and truth. Two things are notable about this perspective.

First, biology is generally pragmatic. Biological systems are tremendously varied, and each is characterized by its own particular details: bone cells are different from brain cells because they express their shared genome in alternate ways and interact with the extracellular environment differently; ants are different from apes because they have their own unique physiology that is determined by their ecological niche and particular ancestry. These intricacies matter and can only be properly understood by meticulous observation and well-defined taxonomies. They do not easily yield to neat theories. Moreover, in many biological contexts, specific details are crucial and are, in fact, more important than principles. In drug discovery, for instance, the precise details of the molecular modes of action of a drug are of utmost importance, not the principles that gave rise to them. Society has benefited tremendously from the focussed pragmatism of generations of meticulous biologists - from medical and pharmaceutical advances, to better understanding of biodiversity and the services that ecosystems provide - and so it should not be disparaged.

However, there is a deeper reason that is less well appreciated. Biologists, like physicists, also have a profound appreciation for the beauty of the natural world. Yet, they often have a more refined sensibility for a different aspect of beauty. To many, beauty is not primarily found in the abstract - in notions such as elegance or simplicity or in mathematical proofs - but rather is found in the extraordinary here-and-now diversity of nature, the "endless forms most beautiful and wonderful" as Darwin described them [7]. Thus, while the physicist may see beauty in simplicity, the biologist may see beauty in interconnection, mutuality and complexity.

No doubt this relates to how the biosciences are taught, which usually places far less emphasis on mathematics than training in the physical sciences. But this is not the only reason. Because they are used to being immersed in the intricacies of the natural world, biologists can be comfortable with the ambiguities and 
complexities of nature in a way that physicists, searching for generalization, might not. Moreover, this immersion elicits a sense of beauty or awe similar to that the physicist feels when encountering an elegant result and so is not just tolerated, it is celebrated. The abundance of nature is beautiful and so is cherished.

\section{Practical consequences}

These are, of course, generalizations: many biologists appreciate elegance; many physicists appreciate intricacy. Nevertheless, it is helpful to articulate these different views, since they have practical consequences.

For example, although there are overarching principles in biology that shape the way that biologists approach their science - the Nobel prize winning biologist, Paul Nurse outlines five such ideas in his recent book, What is Life? [8] - there is sometimes a scepticism of theory, based on a mistrust of abstraction that does not take proper account of biological detail. Many biologists have, in the words of the neurologist Oliver Sacks, "seen grand theories rise, only to be toppled by stubborn facts" [9]. Thus, what the physicist may see as an elegant theory; the biologist may see as a facile generalization.

This objection is not without justification. While often cited as a guide, the principle of simplicity does not have an unambiguous history, and its preeminent place in physics may be due to a romantic view that focusses on examples that prove the rule and excludes those that disprove it. We may be suffering from confirmation bias. In fact, as we gain understanding of the Universe, things tend to get more complex, not simpler, and the mathematical world is also wild [10]. For instance, quantum mechanics and relativity are far more complex than classical mechanics. But they are also much richer - they explain more data and make important predictions, and so provide a deeper understanding of the Universe - and it is this richness that matters. Paul Dirac realized this issue and so had a more nuanced view, suggesting that Einstein's discovery of relativity made it necessary to change the principle of simplicity to one of mathematical beauty [5].

Perhaps more significantly, differing views on beauty have implications for how we approach science and what we consider a good 'explanation'. Alan Turing's theory of morphogenesis is a relevant example. Turing proposed that complex biological patterns might arise when spatially homogeneous states become destabilized by the diffusion of morphogens [11]. Turing-instability offers a straightforward explanation for the generation of a vast array of complex biological phenomena [12]. It is a mathematically beautiful general theory and is recognized as a milestone in our understanding of development [13]. By the criterion of elegance, it is a good 'explanation'.

Yet, despite its power, it does not explain the formation of any particular biological pattern. For that, specific details are needed. Which morphogens, in particular, are involved? How do they react with each other? How are they produced? How do they degrade? How fast do they diffuse? And how do cells respond to them? Answers to these questions are not peripheral: collectively they constitute another good 'explanation' within a specific context, and provide a depth and richness that abstract theory does not. Thus, while it may provide a framework within which to think about patterns in general, Turing's model (or indeed any general model) cannot offer a complete explanation in any particular situation. Indeed, if it is to be validated as a theory of general utility at all, then details must be established in some particular circumstances. Moreover, we now know that a wide variety of mechanisms are also important in establishing and stabilizing biological patterns [14]. Turing's mechanism is one among many. The biological reality is inherently complex: in any given context, principles and specifics are both important and a complete explanation does not consist of either alone, but rather a balanced blend of them both.

\section{The future}

Research at the interface between the physical and biological sciences is hard. This is partly due to well-acknowledged difficulties of cross-disciplinary communication: physicists and biologists use the same terms in different ways, and this can naturally lead to misunderstanding. However, issues of language are relatively easily resolved, given enough time and willingness. I suggest that the primary difficulty in bringing physics and biology together is not differences in language or culture, but what we find attractive. What we 
consider beautiful informs the questions we ask and the answers we consider plausible. Unacknowledged differences at this fundamental level can set us on different scientific trajectories that are hard to unite. Bringing physics and biology together is therefore a particular challenge since it involves bridging two different philosophical approaches to science rooted two different conceptions of beauty.

This does not mean that beauty should be abandoned as a guide: the desire for beauty is arguably an essential part of our humanity and can play an important part in science. Of course, we should be wary of using beauty as our only measure of success. Defining our science solely by what we find attractive risks becoming superficial. If we have different conceptions of beauty, then it also risks becoming subjective and divisive. We must be careful to retain integrity and seek truth with honesty; we must balance beauty with empiricism.

To do this we may need a better appreciation of the many facets of beauty that takes a grander view. One which recognizes that different conceptions of beauty can help illuminate different aspects of life and acknowledges that even apparently superficial notions - such as my basic appreciation of mathematical forms as a child - can be valuable and motivate us to seek deeper truths. This will require that we develop a kind of scientific empathy: the ability to understand and genuinely see another's perspective and thereby learn from them. Those of us that come from the physical sciences can learn to better appreciate beauty in intricacy, interconnection, and complexity and not rush to abstract it away. This is biology's gift to physics. Those of us that come from biological sciences can learn to better appreciate beauty in coherence, elegance, and harmony and accept that behind the apparent complexity of life there may be hidden simplicity. This is physics' gift to biology. Doing this will be good for science and may help us develop a view of life that is richer and more beautiful in every way.

\section{References}

1. Semir Zeki, John Paul Romaya, Dionigi M. T. Benincasa and Michael F. Atiyah, The experience of mathematical beauty and its neural correlates, Front. Hum. Neurosci. (2014)

2. Angela Breitenbach, Beauty in proofs: Kant on aesthetics in mathematics, Eur. J. Philos. 21, 1-23.

3. Richard Feynman, The Messenger Lectures on "The Character of Physical Law", Cornell University November 9, 1964

4. Murray Gell-Mann, Beauty, truth and ... physics?, TED 2007 (https://www.ted.com/talks/murray_gell_mann_beauty_truth_and_physics)

5. Paul Dirac, The relation between mathematics and physics, Proc. Royal Soc. (Edinburgh), 59: 122$129(1939)$

6. John Bernal, The Social Function of Science, George Routledge \& Sons (1939)

7. Charles Darwin, On the origin of species, John Murray, London (1859)

8. Paul Nurse, What is life? Understand Biology in Five Steps, David Fickling Books Oxford (2020)

9. Oliver Sacks, Gratitude, Picador London (2015)

10. Raffaella Mulas, A Survey on Wild Mathematics, arXiv, 2005.01128 (2020)

11. Alan Turing, The chemical basis of morphogenesis, Phil. Trans. Royal. Soc. B, 237 (641) (1952)

12. Shigeru Kondo and Miura Takashi, Reaction-diffusion model as a framework for understanding biological pattern formation, Science 329(5999):1616-1620 (2010)

13. Christopher Surridge, Milestone 4 (1952): Symmetry breaking and computer simulation. Nat. Rev. Neurosci. (2004)

14. Philip Maini and Hans Othmer (Eds.), Mathematical Models for Biological Pattern Formation, Springer, New York, NY (2012) 